\title{
Impact of the DNA methyltransferases expression on the methylation status of apoptosis-associated genes in glioblastoma multiforme
}

\author{
E Hervouet $^{1,2}$, FM Vallette ${ }^{1,2}$ and P-F Cartron ${ }^{\star, 1,2}$
}

Disruption of apoptosis is considered as an important factor aiding tumorigenesis, and aberrant DNA methylation of apoptosisassociated genes could be an important and significant mechanism through which tumor cells avoid apoptosis. However, little is known about (1) the impact of methylation status of apoptosis-associated genes on the presence of apoptosis evasion phenotype in glioma; and (2) the molecular mechanism governing the aberrant methylation of apoptosis-associated genes in glioma. By analyzing human glioma biopsies, we first show that low level of apoptosis in tumor is correlated with aberrant methylation of the bcl-2, bax and XAF-1 genes, but not with the aberrant methylation of the bcl-w, survivin, TMS1, caspase-8 and HRK genes. Our work also indicates that the expression levels of DNA methyltransferase 1 (Dnmt1), Dnmt3b and Dnmt1/Dnmt3a coregulate the methylation status of survivin, TMS1 and caspase-8, whereas no correlation was observed between the expression level of Dnmts and the methylation status of the $b c l-w, b c l-2$, bax, XAF-1 and HRK genes. Thus, these results indicate that the epigenetic regulation of some apoptosis-regulated genes could dictate whether glioma harbors the apoptosis evasion phenotype, and provide some bases to the identification of the methylation machineries of apoptosis-associated genes for which the Dnmt expression acts as a limiting factor.

Cell Death and Disease (2010) 1, e8; doi:10.1038/cddis.2009.7; published online 14 January 2010

Subject Category: Experimental Medicine

This is an open-access article distributed under the terms of the Creative Commons Attribution License, which permits distribution and reproduction in any medium, provided the original author and source are credited. This license does not permit commercial exploitation without specific permission.

Apoptosis or programmed cell death has a crucial role in the maintenance of cellular homeostasis, that is, a balance between cell proliferation and cell death. During tumorigenesis, there is an enhanced ability of the cells to survive in the face of death-inducing mechanisms. Besides, evasion of apoptosis is considered as one of hallmarks characterizing cancer cells. ${ }^{1}$ Cancer cells are known to avoid apoptosis by a number of mechanisms, which include loss of function of tumor suppressor genes though mutations, increased expression of antiapoptotic proteins, expression of oncogenes, increased levels of prosurvival signals, functional inactivation of the members involved in apoptotic signaling, defects in execution pathways of apoptosis and viral-mediated inactivation of tumor suppressor proteins though the interaction of viral protein. ${ }^{1}$

Evidence from literature indicates that DNA methylationmediated regulation of genes involved in apoptosis could be a significant mechanism through which tumor cells avoid apoptosis. ${ }^{2}$ Indeed, evasion of apoptosis can be due to changes in methylation patterns. These changes can occur either in the form of hypomethylation to reactivate antiapoptotic genes or hypermethylation to repress proapoptotic genes. ${ }^{2}$

In the first part of the present report, we have analyzed whether the global DNA methylation status is associated with a phenotype of apoptosis evasion of glioma and whether this phenotype could be explained by the methylation status of eight apoptosis-associated genes. In the second part of this report, we have analyzed whether the methylation status of eight apoptosis-associated genes is directly correlated with the expression levels of DNA methyltransferases (Dnmt), that is, with the expression levels of enzymes catalyzing the DNA methylation of mammalian genes. ${ }^{3,4}$ Indeed, the etiology of aberrant DNA methylation status of apoptosis-regulated genes is not fully elucidated. Except for the participation of Dnmt 1 and of Dnmt3b4/2, a member of the Dnmt3b subfamily of proteins, in the methylation machinery of survivin and RASSF1A genes, no link is clearly established between the methylation of apoptosis-associated genes and Dnmt. ${ }^{3,4}$ Thus, the results of these interrogations could provide the

\footnotetext{
${ }^{1}$ INSERM U892, Centre de Recherche en Cancérologie, Equipe Aspect mécanistiques et physiopathologiques de l'activité des proteines de la famille de Bcl-2 (Equipe labelisée Ligue Nationale Contre le Cancer), 8 quai Moncousu BP70721, 44007 Nantes Cedex 01, France and Faculté de Médecine, Université de Nantes, Nantes, France

${ }^{*}$ Corresponding author: P-F Cartron, INSERM U892, Centre de Recherche en Cancérologie, Equipe Aspect mécanistiques et physiopathologiques de l'activité des proteines de la famille de Bcl-2, 8 quai Moncousu, BP 70721, F-44007 Nantes Cedex 01, France. Tel: + 33228080327 ; Fax: + 3322808 0204; E-mail: pierre-francois.cartron@univ-nantes.fr

Keywords: apoptosis; DNA methylation; glioma; Cancer; Dnmt; GBM

Abbreviations: ChIP, chromatin immunoprecipitation; Dnmt, DNA methyltransferase; GBM, glioblastoma multiforme; TMS1 gene, target for methylation-induced silencing-1

Received 28.10.09; accepted 30.10.09; Edited by P Salomoni
} 
identification of some mechanisms of epigenetic regulation of apoptosis-associated genes responsible, potentially, for the malignant progression of glioma by promoting the appearance of apoptosis evasion phenotype.

\section{Results}

\section{Methylation status of apoptosis-regulated genes in} glioblastoma multiforme. As glioblastoma multiforme (GBM) is known to avoid apoptosis and to present a global DNA hypomethylation pattern, we have searched whether these two parameters can constitute predictive factors of glioma progression. ${ }^{1,5,6}$ For this purpose, we assessed the DNA methylation status through the quantification of 5-methylcytosine $(5 \mathrm{mC})$ number present on DNA using an ELISA method. The phenotype of apoptosis evasion was estimated based on the measure of intra-tumor apoptosis level through quantification of caspase activity (referred to as DEVDase activity), because caspase/DEVDase are final effectors of cell death program. For each parameter, 27 patients were divided into 2 groups based on the $5 \mathrm{mC}$ level or on the level of DEVDase activity found on their tumor biopsies. Survival curves were estimated by Kaplan-Meier method and compared by using the Cox proportional hazards survival regression analysis. Thus, significant difference was observed between patients whose tumors had high level of $5 \mathrm{mC}$ and those whose tumors did not, and between patients whose tumors had high level of DEVDase activity and those whose tumors did not $(P=0.0113$ and $P=0.0466$, respectively) (Figure $1 \mathrm{a}$ and $\mathrm{b}$ ). In other terms, these results indicate that the low level of $5 \mathrm{mC}$ and the low level of DEVDase activity are two poor prognosis factors.

We then continued our study by searching whether these two parameters are correlated. A statistical analysis using Pearson's correlation test showed a significant correlation between these two parameters in GBM: low DEVDase activity is correlated with low $5 \mathrm{mC}$ number $(r=0.4161, P=0.0309)$ (Figure 1c). Moreover and as expected, Kaplan-Meier and Cox proportional hazards survival regression analyses associated the copresence of low $5 \mathrm{mC}$ number and low DEVDase activity in tumors with the weak survival of patients whose tumors had these two hallmarks (Figure 1d). Thus, all these results suggest that the DNA methylation could have a crucial role in the disruption of execution of cell death program in GBM. Consistently, some reports have already described that apoptosis-associated genes can be epigenetically regulated in glioma. ${ }^{7-13}$

To study the impact of methylation-induced disruption of the execution of apoptosis, we have decided to examine the methylation status of eight genes encoding for proteins regulating the apoptotic program in a collection of $27 \mathrm{GBM}$ patients (Figure 2a). The eight genes chosen were (1) caspase- 8 gene encodes for an initiator caspase, which is characterized by the presence of two homotypic interaction motifs called DEDs (death-effector domains). Caspase-8 cleaves Bid to generate truncated Bid, which activates proapoptotic proteins Bax and Bak to promote apoptosis through the cytochrome $c$ release from the mitochondria and caspase-3 activation. Caspase-8 can also directly activate effector caspases such as caspase-3; (2) TMS1 gene (target for methylation-induced silencing-1 or apoptosis-associated speck-like protein containing CARD - ASC) encodes for a protein consisting of PYD (pyrine domain) and CARD motifs. TMS1/ASC has been shown to induce apoptosis in a caspase-8-dependent manner; ${ }^{14}$ (3-4) bcl-w and bcl-2 genes encode for two antiapoptotic proteins characterized by the presence of all $\mathrm{BH} 1-4$ domains. Antiapoptotic proteins block apoptotic program by inhibiting the proapoptotic proteins such as Bax; ${ }^{15}$ (5) HRK gene encodes for the proapoptotic protein HRK (harakiri), which belongs to the $\mathrm{BH} 3$-only protein family and selectively antagonizes the antiapoptotic proteins Bcl-2 and $\mathrm{Bcl}-\mathrm{xl} ;{ }^{16}$ (6) bax gene encodes for the p21 bcl-associated $\mathrm{X}$-protein or $\mathrm{Bax} \alpha$. Bax $\alpha$ induces the release of cytochrome $c$ from the mitochondria, activation of caspase-3 and thereby apoptosis; ${ }^{17}$ (7) survivin gene encodes for an antiapoptotic protein characterized by the presence of the Baculoviral inhibitor of apoptosis protein repeat (BIR) motif, which also characterized the Inhibitor of Apoptosis Proteins (IAPs). Survivin can inhibit apoptosis by binding and blocking the activation of caspase- $3 ;^{18}$ (8) XAF-1 gene encodes for the $X$ linked inhibitor of apoptosis-associated factor-1 (XAF-1). $X A F-1$ negatively regulates $X$-linked inhibitor of apoptotsis (XIAP), a member of the IAP family (XIAP, c-IAP-1 and c-IAP2), which are potent inhibitors of caspase-3, -7 and $-9 .^{19}$

Methylation-specific PCR (MSP) was used to determine the methylation status of these eight genes, according to the primers designed by using the MethPrimer program (Supplementary Figure S1). ${ }^{20}$ With respect to the bax gene, we distinguished two methylation profiles. The first, named bax ${ }^{B a x} \psi$, is associated with Bax $\psi$ expression, and the second, referred to as bax silencing, is correlated with the bax gene silencing. ${ }^{7,8}$ MSP showed that methylation of $b c l-w, b c l-2$, bax ${ }^{\text {silencing }}$, bax ${ }^{\text {Bax }}$, survivin, XAF-1, TMS1, caspase-8 and HRK genes occurs with a frequency of $19,22,4,19,30,22,48$, 30 and $30 \%$ in our collection of 27 GBM patients (Figure $2 b$ ).

On the basis of these results, we next determined whether the methylation of these genes confers a poor prognosis in GBM patients. Survival curves were estimated by KaplanMeier method and compared by using the Cox proportional hazards survival regression analysis. Thus, significant difference was observed between patients whose tumors had the $\operatorname{bax}^{\mathrm{BAX} \psi}$ methylation profile and those whose tumors did not $(P=0.0044)$, whereas no significant difference was observed for the methylation profile of other considered genes $(\mathrm{bcl}-\mathrm{w}$, $P=0.6690, b c l-2, P=0.3437$; survivin, $P=0.4328 ; X A F-1$, $P=0.2986 ;$ TMS1, $P=0.4379$; caspase-8, $P=0.116$ and $H R K, P=0.2759)$ (Figure $2 \mathrm{c}$ ). The presence of the bax silencing methylation profile confers short survival time to the patient harboring this hallmark (3.1 months). However, this point must be confirmed with more samples.

We then complemented this study by analyzing whether the unmethylation of genes coding for proapoptotic proteins and the methylation of genes coding for antiapoptotic proteins could collectively confer a good prognosis for GBM patients. For this purpose, we calculated the score of methylation of apoptosisassociated genes. A value of +1 was attributed to each tumor harboring the methylated gene coding for an antiapoptotic protein or the unmethylated gene coding for a proapoptotic protein. A value of -1 was attributed to each tumor harboring the unmethylated gene coding for an antiapoptotic protein or the 

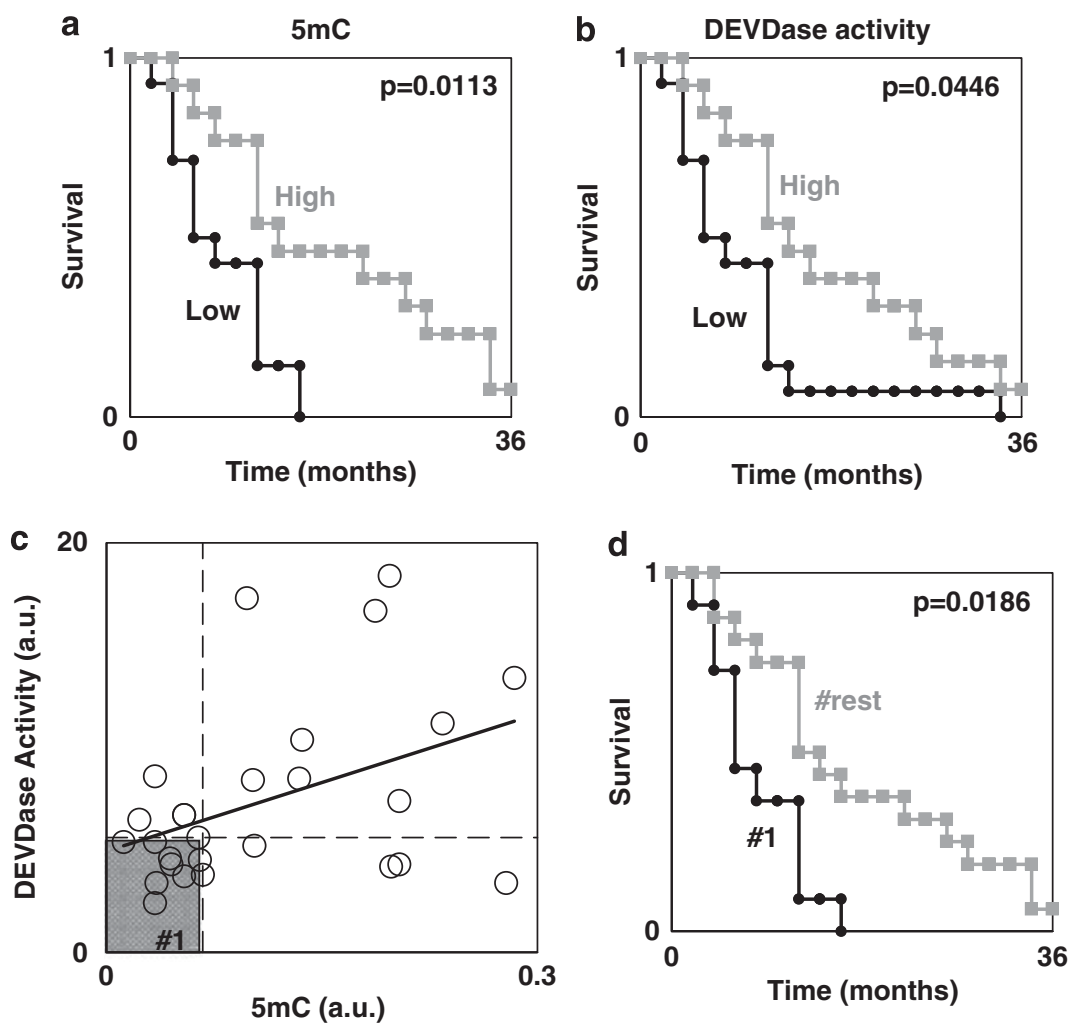

Pearson's correlation test
$r=0.4161, p=0.0309$

Figure 1 5-methylcytosine $(5 \mathrm{mC})$ number and the DEVDase activity in human GBM. $(\mathbf{a}, \mathbf{b})$ Kaplan-Meier estimates of time to survival in function of $5 \mathrm{mC}$ number or of level of DEVDase activity. The $5 \mathrm{mC}$ number was assessed with the Methylamp Global DNA methylation quantification kit (Euromedex-Epigentek). DEVDase activity, representing the activation level of the main effective caspases of apoptosis, was measured as previously described. ${ }^{37}$ High: patients whose tumors are characterized by a $5 \mathrm{mC}$ level or a DEVDase activity higher than the median; low: patients whose tumors are characterized by a $5 \mathrm{mC}$ level or a DEVDase activity equal to or lower or than the median. (c) Correlation between the $5 \mathrm{mC}$ and the DEVDase activity in human GBM. Black area (\#1) regroups patients whose tumors are characterized by the copresence of a low $5 \mathrm{mC}$ number and a low level of DEVDase activity. (d) Kaplan-Meier estimates of time to survival in function of the copresence or not of a low $5 \mathrm{mC}$ number and a low level of DEVDase activity in glioma. \#1 represent the group of patients whose tumors are characterized by the copresence of a low $5 \mathrm{mC}$ number and a low level of DEVDase activity

methylated gene coding for a proapoptotic protein. With respect to the bax gene, +1 was attributed when the $\operatorname{bax}^{\mathrm{BAX} \psi}$ methylation profile was observed, and -1 was attributed when the bax silencing methylation profile was observed. Thus, 11 tumors had a positive score (group no. A), that is, a score potentially associated with the responsiveness of apoptosis, whereas 16 tumors harbored a negative or null score (group no $\mathrm{B})$, that is, a score potentially associated with the apoptosis escape phenotype. Besides, these points were supported by the presence of a significant correlation existing between the methylation score and the DEVDase activity of each tumors (Figure 2d). Survival curves of these two groups were estimated by Kaplan-Meier method, and the Cox proportional hazards survival regression analysis revealed that the presence of negative or null score of methylation status of the considered apoptosis-associated genes confers poor prognosis of GBM patients (Figure $2 \mathrm{~d}$ ).

All these results confirm that the presence of the bax ${ }^{\mathrm{BAX}} \psi$ methylation profile is associated with a good prognosis in GBM patients, and show that the methylation score of apoptosis-associated genes can also confer a good prognosis in GBM patients, despite the fact the methylation status of these genes is not individually a prognosis factor. ${ }^{7}$
Low level of DNA methylation was associated with the unmethylation of bcl-w, bcl-2 and survivin genes. We next complemented our study by analyzing whether the methylation status of these genes was correlated with the global DNA methylation status. For this purpose, the $5 \mathrm{mC}$ number and the methylation status of each gene were plotted against each other, and statistical analyses using the Pearson's correlation test showed a correlation between the low level of $5 \mathrm{mC}$ number on DNA and the unmethylation of the bcl-w, bcl-2 and survivin genes $(r=0.5507$, $P=0.0029 ; r=0.7521, P<0.0001 ;$ and $r=4467, P=0.0195$, respectively) (Figure 3 ). These findings suggests that the unmethylation of antiapoptotic genes, such as $b c l-w, b c l-2$ and survivin, occurring in a context of low genome methylation could be because of many molecular causes conferring the apoptosis evasion phenotype seen in glioma.

The bcl-2 gene unmethylation and/or the methylation of XAF-1 and bax ${ }^{\text {Bax } \psi}$ genes influence the intra-tumor apoptosis level. Consistent with the previous hypothesis, we next investigated whether the methylation status of these genes was correlated with the levels of intra-tumor apoptosis. Thus, the methylation status of each gene and DEVDase 
a
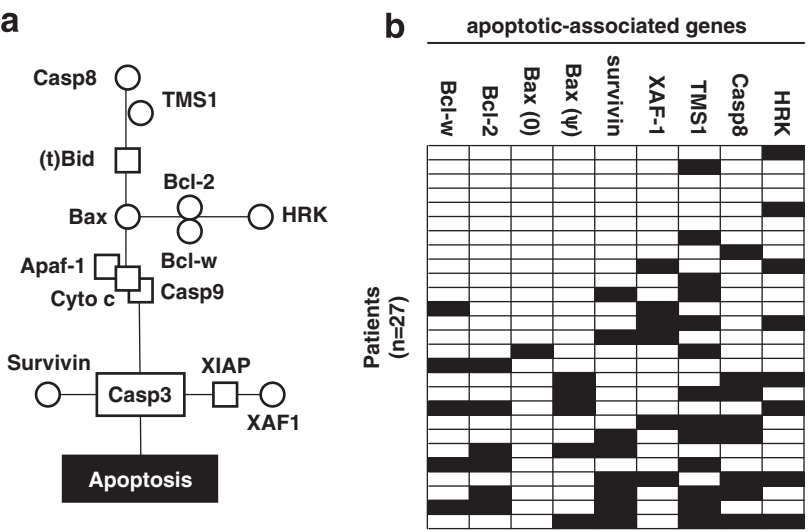

c

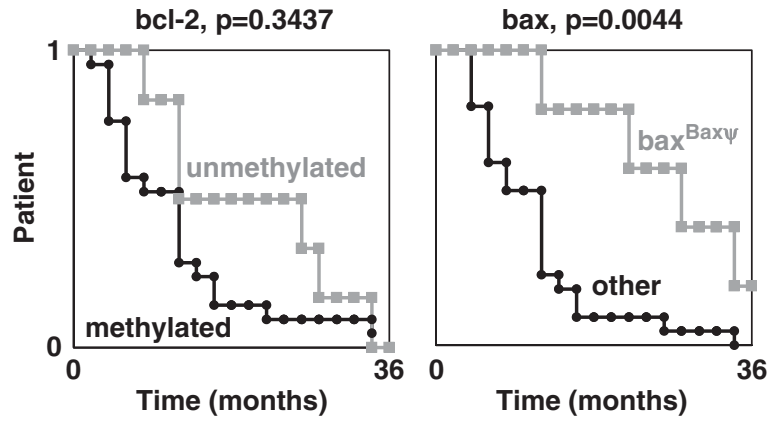

d Pearson's correlation test
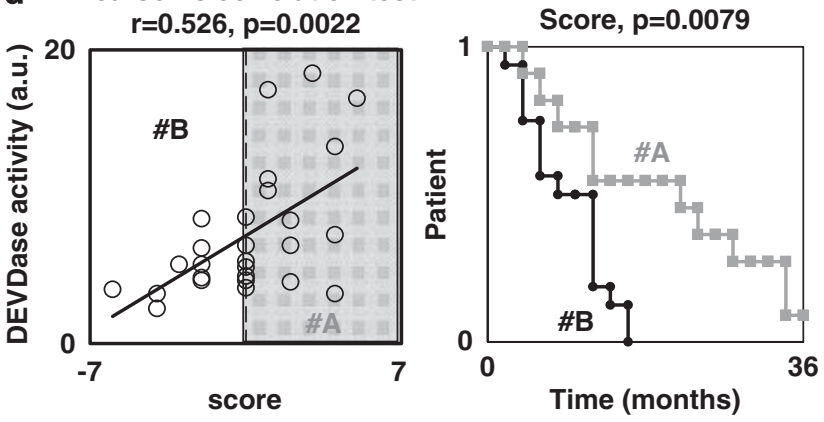

Figure 2 Impact of the methylation status of the HRK, XAF-1, survivin, bcl-w, TMS1, bcl-2, caspase-8 and bax genes on survival time of patients with GBM. (a) Schematic representation of apoptotic pathway. (b) Methylation status of the HRK, XAF-1, survivin, bcl-w, TMS1, bcl-2, caspase-8 and bax genes in 27 human GBM patients. Open box represent unmethylated gene and black box represents methylated gene. (c) Kaplan-Meier estimates of time to survival of GBM patients in function of the methylation status of the $b c l-2$ or bax genes. (d) Left: correlation between the methylation score of the apoptosis-associated genes and the DEVDase activity. Group \#A defines tumors having a positive score, that is, a score potentially associated with the responsiveness of apoptosis; group \#B defines tumors harboring a negative or null score, that is, a score potentially associated with the apoptosis escape phenotype. Right: Kaplan-Meier estimates of time to survival in function of the membership of group \#A or \#B

activity were plotted against each other, and statistical analysis using the Pearson's correlation test was performed to determine the presence of a significant correlation between these parameters. As illustrated by the Figure $4 \mathrm{a}$ and Supplementary Figure S2, we noted that the methylation of $b c l-2$ gene was correlated with the presence of high DEVDase activity in GBM $(r=0.3866 ; P=0.0464)$, whereas no correlation was observed between the methylation of the $b c /-w$ or survivin genes and the DEVDase activity
( $r=0.1721, P=0.3907$ and $r=0.0468, P=0.8167)$. Similar experiments showed the presence of a close correlation between the presence of the bax ${ }^{B a \psi}$ methylation profile and high level of DEVDase activity in GBM ( $r=0.8047$; $P<0.0001)$. The presence of methylated $X A F-1$ gene was correlated with the presence of low DEVDase activity in GBM $(r=0.3920 ; P=0.0431)$. Although not being correlated with the DEVDase activity, we noted that the presence of the bax ${ }^{\text {silencing }}$ gene occurred in GBM presenting the lower level of DEVDase activity. Finally, our study showed no correlation between the methylation status of TMS1, caspase-8 or HRK genes and the level of intra-tumor DEVDase activity $(r=-0.2525, \quad P=0.2039 ; \quad r=0.2008, \quad P=0.3152 ; \quad r=$ $-0.1796, P=0.3700$; and $r=0.1804, P=0.3679)$. We then complemented these data by observing that the methylation of the $b c /-2, b a x^{\text {silencing }}$ and $X A F-1$ gene was correlated with the silencing or the low expression of these genes (Figure 4b). Taken together, these results suggest that the methylation-dependent silencing or downregulation of $b c l-2$, bax and $X A F-1$ genes has a crucial role in the execution of apoptotic program in GBM.

To confirm this point, we decided to mime the methylationdependent silencing or downregulation of $b c /-2$, bax and $X A F-1$ genes by performing gene invalidation experiments. The efficiency of invalidations has been monitored by western blots, and programmed cell death was estimated by DEVDase activity. Consistent with what we have already published, we first noted that the invalidation of Bax was a sufficient point to promote resistance of apoptosis in glioma cells previously sensitive to etoposide-induced apoptosis (Figure 4c). ${ }^{8}$ Similarly, our results indicated that siRNA-induced XAF-1deficiency was sufficient to generate a low level of apoptosis in glioma cells (Figure 4d). According to the antiapoptotic role of $\mathrm{Bcl}-2$, we noted that the shRNA-induced invalidation of $b c l-2$ gene expression was sufficient to increase the etoposideinduced apopto-sensitivity of glioma cells (Figure 4e). Thus, our data indicate that the loss or the weak expression of the $b c l-2$, bax or XAF-1 genes induced by their aberrant methylation or by their invalidation is due to many conditions influencing the execution of apoptosis.

Correlation between the methylation status of apoptosisregulated genes and the Dnmt expression. To establish a relation between the methylation status of the apoptosisregulated genes included in our study and the Dnmt expression in GBM, we complemented our study by analyzing the level of expression of Dnmt1, Dnmt3a and Dnmt3b by ELISA, because these three Dnmts are the main enzymes implicated in DNA methylation in mammals. Pearson's correlation test revealed that the methylation status of caspase-8 gene was correlated with the Dnmt1 and Dnmt3a expressions (Figure 5). Similar statistical analyses also indicated that the methylation status of survivin was correlated with Dnmt1 expression, whereas the methylation status of TMS1 was correlated with the Dnmt3b expression (Figure 5). No relevant correlation was revealed by the Pearson's correlation test in our collection of 27 GBM patients between the Dnmt expression and the methylation status of $b c l-w, b c l-2, \quad b a x, \quad X A F-1$ and $H R K$ genes (Supplementary Figure S3). 

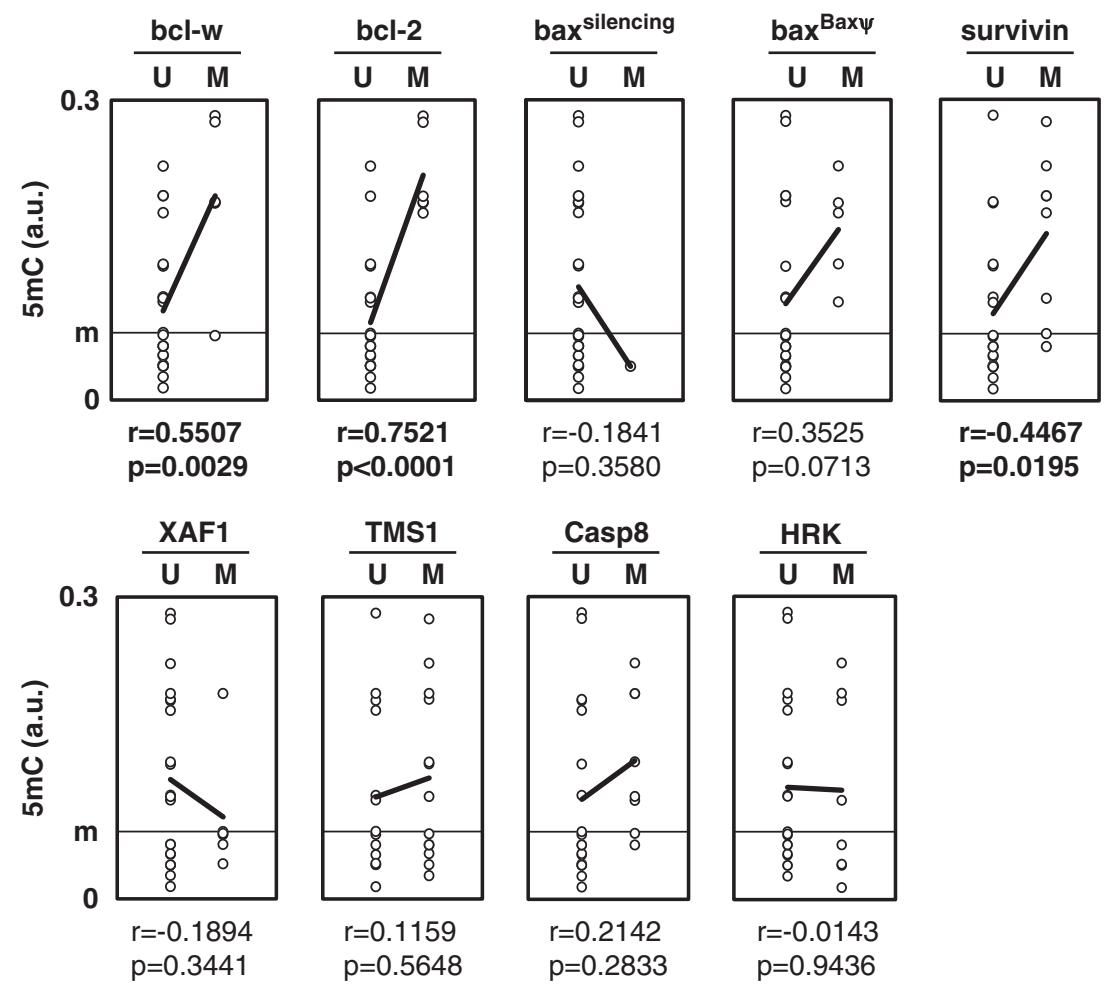

Figure 3 Correlation between the 5-methylcytosine number $(5 \mathrm{mC})$ and the methylation status of the HRK, XAF-1, survivin, bcl-w, TMS1, bcl-2, caspase-8 and bax genes. $\mathrm{U}$, unmethylated; $\mathrm{M}$, methylated; $r$, correlation coefficient obtained from Pearson's correlation test; and $P$, significance of a correlation coefficient

To confirm the role had by Dnmt3b on the establishment of the methylation status of TMS1 gene, we first analyzed whether the overexpression of the enzyme could enhance the methylation of these genes through the de novo methylation property of these enzymes. Comparing the U87 cells with the U87 cells treated with pORF-Dnmt3b, we observed an increase in the methylation status of TMS1 gene, whereas the methylation of caspase-8 was unchanged under this condition (Figure 6a and Supplementary Figure S4). After transformation of the U87 cells with the pORF-Dnmt3a, we noted an increase in the methylation status of caspase-8 gene, without change in the methylation status of TMS1 gene (Figure 6b).

In parallel with these results, we also decided to confirm the correlation obtained from GBM by analyzing the impact of Dnmt invalidation on the methylation status of caspase-8, survivin and TMS1 genes in U251 cells. Dnmt invalidation was monitored by qPCR and the methylation status of caspase-8, survivin and TMS1 genes was monitored by Methylation Array and Methylated DNA Immunoprecitation (MeDIP) (Supplementary Figure S5).

We thus noted that the single Dnmt1 invalidation in U251 cells abolished the methylation of caspase- 8 gene and that the single Dnmt3a invalidation decreased the methylation status of this gene (Figure 6c). As expected, the single invalidation of Dnmt1 affected the methylation status of survivin gene by inducing a significant hypomethylation (Figure 6d). Consistent with our previous results, we observed that the invalidation of Dnmt3b abolished the methylation status of TMS1 gene (Figure 6e). Taken together, these results indicate that the methylation machinery of TMS1 gene includes the Dnmt3b, that one of the survivin gene includes the Dnmt1 and that the one of caspase- 8 gene includes the cooperation of Dnmt1 and Dnmt3a.

\section{Discussion}

Cancer cells are known to harbor aberrant DNA methylation profiles (global DNA hypomethylation and gene-specific hypoor hypermethylation) and defects in apoptotic pathway. In this study, we observed that the loss of apoptosis is correlated with the loss of DNA methylation in GBM. At molecular level, the analysis of a collection of 27 GBM patients revealed that the unmethylation of $b c l-2$ gene is the only hallmark associated with a low level of genome methylation in the one hand and with a low intra-tumor apoptosis level in the other hand. In other terms, our study suggests that the unmethylationinduced overexpression of $\mathrm{Bcl}-2$ is a factor aiding the GBM to acquire the apoptosis evasion phenotype in a context of global DNA hypomethylation. Except for the methylation status of $b c l-2$ gene, we noted that the unmethylation of the $b c l-w$ and survivin genes is also correlated with a low DNA methylation level. Besides, there is a strong association between the methylation status of the $b c l-w$ and $b c l-2$ genes, which were correlated in $89 \%$ of cases ( $r=0.6625, P=0.0002)$, whereas there is no correlation between the methylation status of these two genes and the methylation status of survivin gene ( $r=-0.1005, P=0.6179$ and $r=0.2385, P=0.2309$ ). Thus, it seems that the methylation machineries controlling the methylation status of survivin gene in the one hand and the methylation status of the $b c l-w$ and $b c l-2$ genes in the other hand are distinct. This idea is also supported by the fact that the methylation status of survivin gene is correlated with the 

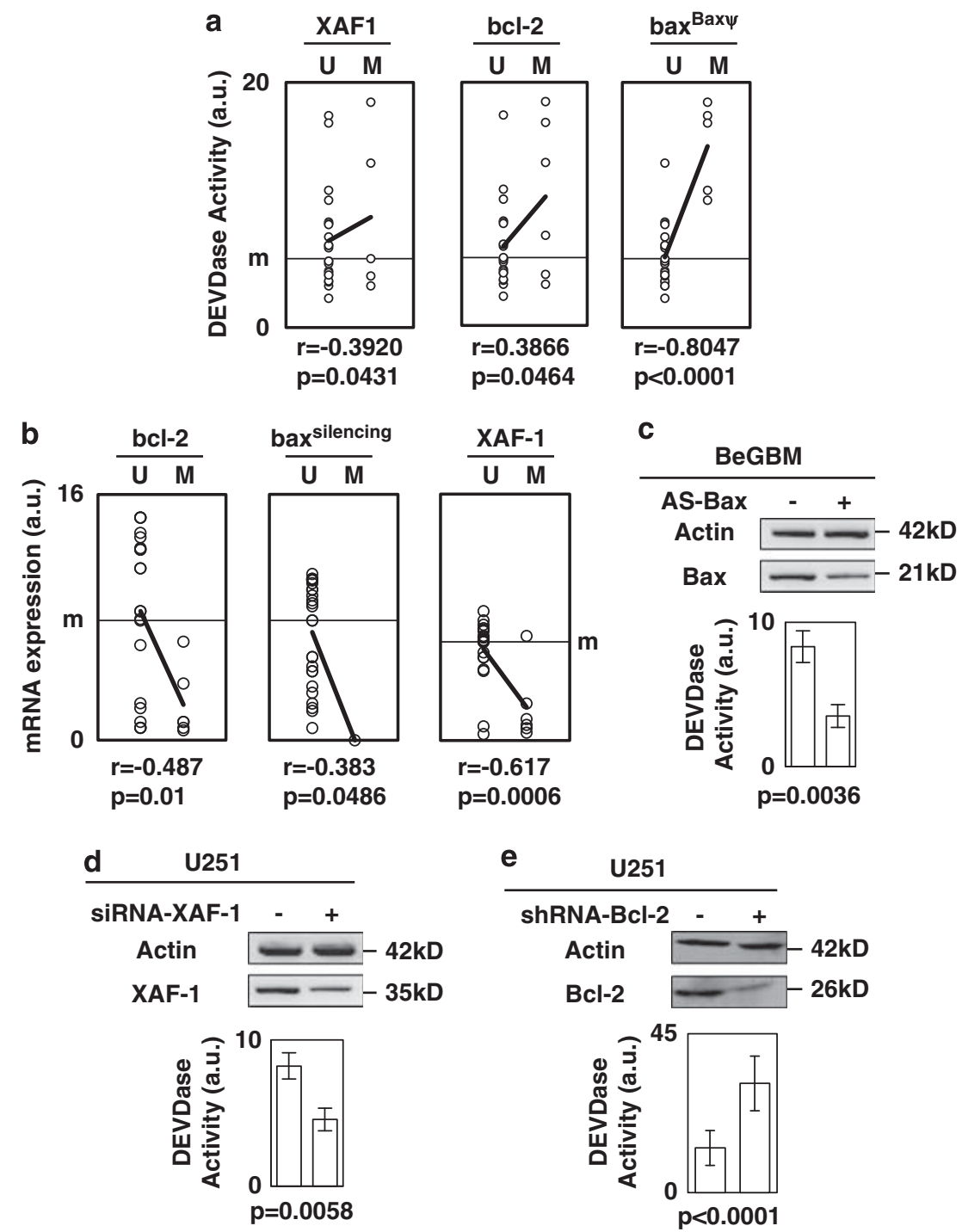

Figure 4 Impact of methylation status of apoptotic-associated genes on apoptosis. (a) Correlation between the DEVDase activity and the methylation status of the XAF-1, bcl-2 and bax genes. U, unmethylated; M, methylated; $r$, correlation coefficient obtained from Pearson's correlation test; and $P$, significance of a correlation coefficient. (b) Correlation between the bax ${ }^{\mathrm{mRNA}}$, bcl-2 ${ }^{\mathrm{mRNA}}$ and XAF- ${ }^{\mathrm{mRNA}}$ expression levels and the methylation status of the bax, bcl-2 and XAF-1 genes. U, unmethylated; M, methylated; $r$, correlation coefficient obtained from Pearson's correlation test; and $P$, significance of a correlation coefficient. (c-e) Impact of invalidation of the bax, bcl-2 or XAF-1 genes on apoptosis in glioma cells. BeGBM, Bax-expressing GBM. Western blots were carried out to confirm the gene invalidations, and DEVDase activity was assessed $24 \mathrm{~h}$ after apoptosis induction by etoposide treatment $(50 \mu \mathrm{g} / \mathrm{ml})$

Dnmt1 expression, whereas no correlation between the Dnmt expression and the methylation status of the $b c /-w$ and $b c /-2$ genes was seen. Further, our observation is consistent with the one published by Esteve et al., ${ }^{21}$ according to which Dnmt1 participates in the survivin gene regulation. In addition, the fact that the unmethylation of survivin, $b c l-w$ or $b c l-2$ genes is correlated with the low level of global DNA methylation suggests that the methylation machineries regulating the methylation of these genes is also implicated in the loss of global DNA methylation, or that the loss/dysfunction of methylation machineries controlling the level of global DNA methylation and the methylation status of specific genes, such as survivin, $b c l-w$ and/or $b c l-2$, are regulated by shared/ common ethiological factors. As the methylation of caspase-8 gene is correlated with the expression level of Dnmt1 and Dnmt3a, our data suggest that both Dnmts cooperate to promote and/or maintain the methylation of caspase- 8 gene. To date, functional cooperation between Dnmt1a and Dnmt3a has been already described for DNA and for repetitive elements, but not for caspase-8 gene. ${ }^{22-24}$ Besides, the cooperation between Dnmt1 and Dnmt3a in the methylation machinery of caspase- 8 gene is one of the first examples of the Dnmt1-Dnmt3a cooperation to methylate a specific gene, according to our knowledge. Nevertheless, the cooperation between de novo and maintenance methyltransferases to methylate a specific gene has already been described. Thus, the literature reports that Dnmt1 and Dnmt3b cooperate to methylate specific genes, such as $p 16^{\text {INK4a } I g f 2, ~ B A G 1 ~ o r ~}$ CXCL12, for example. ${ }^{25-27}$ Our work also associates the Dnmt3b expression level with the methylation status of the TMS1 gene. Despite the absence of Dnmt identification, Stimson et al. ${ }^{28}$ report that the methylation-mediated silencing 


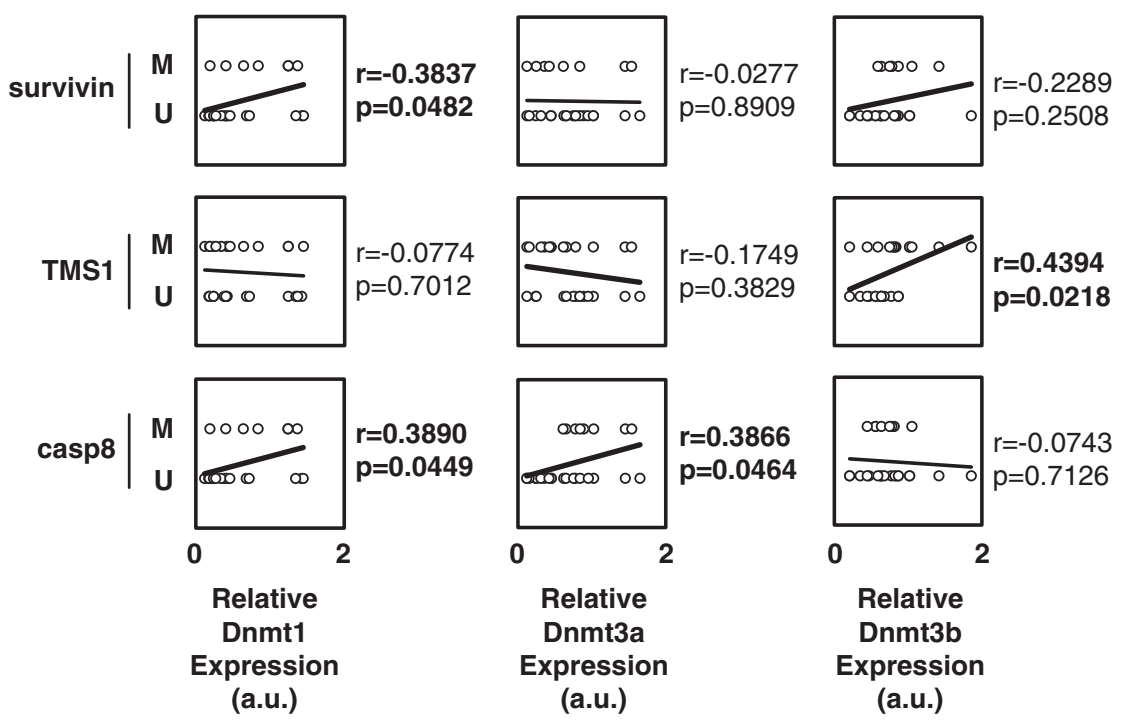

Figure 5 Correlation between the Dnmt expression and the methylation status of apoptotic-associated genes. The expression of Dnmt was assessed by ELISA method using EpiQuik Dnmt1 Assay Kit, EpiQuik Dnmt3a Assay Kit and EpiQuik Dnmt3b Assay Kit (Euromedex). U, unmethylated; M, methylated; $r$, correlation coefficient obtained from Pearson's correlation test; and $P$, significance of a correlation coefficient

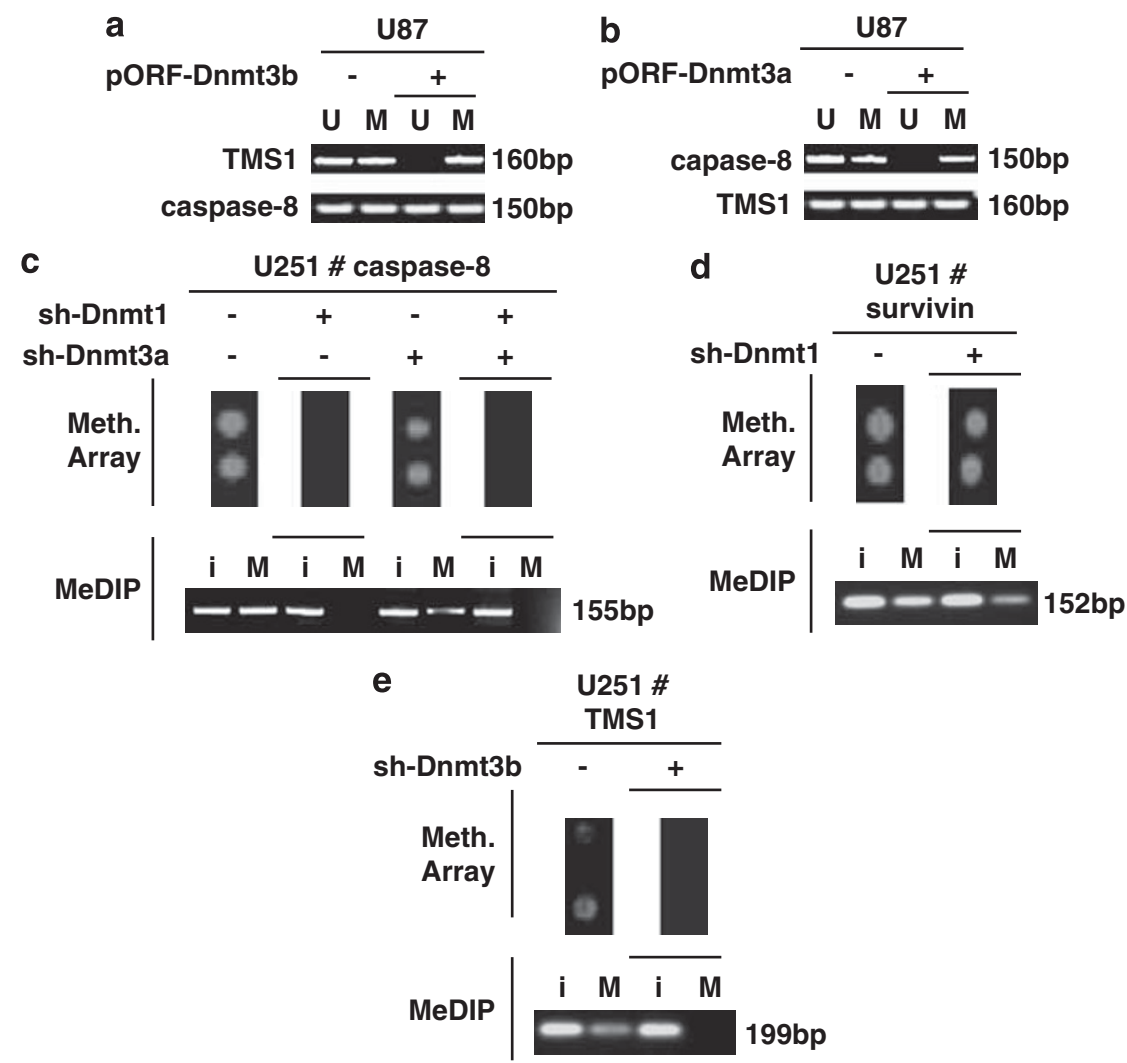

Figure 6 Impact of Dnmt overexpression or invalidation on methylation status of the caspase-8, TMS1 and survivin genes. (a and $\mathbf{b})$ De novo Dnmt3a and Dnmt3b are overexpressed in U87 cells and the methylation status of the TMS1 and caspase-8 genes was investigated by methylation-specific PCR (MSP). U, unmethylated; M, methylated. (c) Impact of Dnmt1 and/or Dnmt3a invalidation on the methylation status of caspase-8 gene in U251 cells illustrated by the results obtained in methylation array and methylated DNA immunoprecipitation (MeDIP). (d) Impact of Dnmt1 invalidation on the methylation status of survivin gene in U251 cells, illustrated by the results obtained in methylation array and MeDIP. (e) Impact of Dnmt3b invalidation on the methylation status of TMS1 gene in U251 cells illustrated by the results obtained in methylation array and MeDIP 
of TMS1 gene is accompanied by histone hypoacetylation, suggesting that Dnmts that are able to interact with a HDAC protein can potentially promote the methylation of TMS1 gene. Thus, our data showing the fact that Dnmt3b is a factor of the TMS1 methylation machinery are consistent with this idea and also with the fact that Dnmt3b can interact with HDAC. $^{29}$ Further, Supplementary data confirm this point by showing that the methylation of TMS1 gene in the U87 and U251 cells was associated with the corecruitment of Dnmt3b, HDAC1 and Dnmt1 on the TMS1 promoter (Supplementary Figure S6). Thus, this result obtained by chromatin immunoprecipitation is interesting for several reasons. First, it indicates that the TMS1 methylation machinery also implicates a cooperation of Dnmt1 and Dnmt3b despite the absence of correlation between the expression level of Dnmt1 and the methylation status of TMS1 gene. Second, this result confirms the idea that Dnmt1 is a factor of the TMS1 methylation machinery. ${ }^{30}$ Third, these results, combined with our previous data, show that the Dnmt1 expression level is not a limiting factor for the TMS1 gene methylation machinery in GBM, contrary to the situation seen in fibroblast, in which TMS1 gene is aberrantly methylated and silenced in response to the Dnmt1 overexpression. ${ }^{30}$

Thus, all the results presented above provide some bases to the identification of the methylation machineries of apoptosis-associated genes, for which the Dnmt expression act as a limiting factor.

With regard to the impact of the eight considered apoptoticassociated genes on the intra-tumor apoptosis level, our experiments show that only the methylation status of $b c /-2$, bax and $X A F-1$ genes can influence the intra-tumor apoptosis level. Consistent with our previous publications, we here confirm that bax gene can be methylated on its promoter part only or on its promoter part and on its intronic1 region. ${ }^{7,8}$ Thus, the first methylation profile is correlated with high apoptosis level in GBM, because it encodes for Bax $\psi$ expression, an isoform more apoptogenic than wild-type Bax named Bax $\alpha$. The second methylation profile is associated with weak apoptotic level in GBM, because it promotes the bax silencing in glioma cells. Moreover, the presence of several methylation profiles of bax gene in GBM is interesting in terms of the understanding of DNA methylation mechanisms for several reasons. First, this can suggest the presence of distinct methylation machineries: one able to promote the methylation of bax promoter region and a second able to promote the methylation of bax intronic1 region. Second, according to this hypothesis, it is potentially possible that the bax silencing methylation profile can result from the cooperation between these two methylation machineries to promote the bax silencing methylation profile from the $b_{a x}{ }^{\mathrm{Bax} \alpha}$ methylation profile. Third, the fact that the bax $x^{\text {silencing }}$ methylation profile corresponds to the $b a x^{\mathrm{Bax} \psi}$ methylation profile added with the methylation of bax intronic1 region can suggest that the bax silencing methylation profile can result from methylation of $b a x^{\mathrm{Bax} \psi}$ methylation profile. A study of these hypotheses applied to a greater sample size is currently in progress in our lab. As already discussed above, our analysis reveals a correlation between the $b c l-2$ gene methylation status and the level of intra-tumor apoptosis in GBM. This point is probably due to the fact that the $b c /-2$ gene methylation status is correlated with the level of $b c /-2$ gene expression in our collection of 27 GBM patients. Indeed, several authors report that the methylation status of $b c l-2$ gene did not necessarily correlate with the relative levels of $\mathrm{Bcl}-2$ expression. ${ }^{31,32}$ Thus, these finding suggest that the $b c l-2$ gene methylation may be the one, but probably not the essential, mechanism regulating the expression of this gene. Besides, this point is an ongoing subject of study in our lab. Finally, the presence of correlations between the $X A F-1$ gene methylation status, the expression level of $X A F-1$ gene and the level of intra-tumor apoptosis in GBM is consistent with similar observations obtained from several cancer types such as renal cell carcinoma, human gastric and colon cancer, for example. ${ }^{33-35}$

In conclusion, our study indicates that the aberrant epigenetic regulation of the apoptosis-associated genes contributes to the malignant progression of human tumors by aiding the appearance of apoptosis evasion phenotype and by conferring poor prognosis in patients with glioma. Although our data identified the variations of Dnmt expression as proofs to explain the presence of $3 / 8$ aberrant DNA methylation profile of apoptosis-associated genes, we cannot exclude the fact that the presence of 5/8 aberrant DNA methylation profile of apoptosis-associated genes is not explained by the variations in Dnmt expression. This last point introduces the idea that the Dnmts, despite their central role in the DNA methylation machinery, work in a network of proteins working in concert, the characterization of which will be a new step in the understanding of molecular mechanisms promoting the resistance of apoptosis in glioma.

\section{Materials and Methods}

Patient characteristics. Overall survival was measured from the date of surgical resection to the death. All patients included in this study had similar management (complete resection) and similar treatment (temozolomide and fractionated radiotherapy). Karnofsky performance scores of patients are included in the $80-90$ range and age is included in the $49-65$ years range.

Epigenetic analysis. DNA was extracted by using the QiaAmp DNA mini Kit (Qiagen, Courtaboeuf, France). The $5 \mathrm{mC}$ number was assessed with the Methylamp Global DNA methylation Quantification kit (Euromedex-Epigentek, Strasbourg, France). The methylation status of apoptotic-associated genes was determined by MSP, according to the primers designed by using the MethPrimer ${ }^{38}$ program and after sodium bisulfite conversion (EZ DNA methylation Gold kit, Zymo research, Orange, CA, USA). MeDIP were performed by using MeDIP kit according to the manufacturer's instructions (Diagenode, Evry, France). Primers used in this study were caspase-8, s: 5'-GGTGGAGGTTGCAGTGAG-3', as: 5'-TGGA GTGTTGGTGCTACTGA-3'; TMS1, s: 5'-CCCCAGTATGTGGAATTGAGG-3', as: $5^{\prime}$-TTGCACCAGCGGGTACAGA-3'; survivin, s: 5'-AGTAGAGACAAGGTTTCACC G-3', as: 5'-GCCCCCTCGACTGCTTT-3'.

Western blot. In brief, $50 \mu \mathrm{g}$ of proteins were size fractionated by sodium dodecyl sulfate polyacrylamide gel electrophoresis. Proteins were transferred onto nitrocellulose or PVDF membrane. Saturation and blotting were realized by using SNAP i.d Protein Detection System (Millipore, Molsheim, France). The detection of proteins was carried out using ECL (Amersham Biosciences, Pantin, France) and/or SuperSignal west femto maximum sensitivity (Pierce, Thermo Fisher Scientific, Brebiéres, France) chemiluminescence reagents. Bands were quantified using Quantity One quantification software (BioRad, Marnes-la-coquette, France). AntiBax (Millipore, no. 06-499); anti-Bcl-2 (Epitomics, Euromodex, Mundolsheim, France, no. 1018-1); anti-actin (Chemicon, Hants, Great Britain, no. MAB1501R); and anti-XAF-1 (Abcam, Paris, France, no. 17204).

Quantitative PCR. RNA was isolated from cells using trizol. Quantitative PCR (qPCR) reactions were done in triplicate by using the Multiplex Quantitative 
PCR system (Mx4000), qPCR Core Reagent kit (Stratagene, Agilent, Masy, France), according to manufacturer's instructions, and by using primers specific for Bax (F: $5^{\prime}$-GCTGTTGGGCTGGATCCAAG-3' ${ }^{\prime}$ and R: $5^{\prime}$-TCAGCCCATCTTCTT CCAGA- $3^{\prime}$ ) and Bcl-2 (F: $5^{\prime}$-ATGTGTGTGGAGAGCGTCAACC-3' and R: $5^{\prime}$-TG AGCAGAGTCTTCAGAGACAGCC- $3^{\prime}$ ). XAF-1 ${ }^{\mathrm{mRNA}}$ expression was assessed by using primers designed by Fong et al..$^{36} \mathrm{GAPDH}$ was used as housekeeping gene (F: 5'-GTGAAGGTCGGAGTCAACG-3' and R: 5'-TGAGGTCAATGAAGGGGTC-3')

Caspase activation assay. Total cell lysis was carried out and protein concentrations were measured using the Bradford technique. DEVDase activity was measured using the fluorometric CaspACE Assay System (Promega, Paris, France) and normalized to the sample protein concentration, as described previously. ${ }^{37}$

Gene invalidation experiments. XAF-1 gene was invalidated by using XAF-1 siRNA (Santa Cruz, Heildelberg, Germany, sc-37511) according to manufacturers instructions. Bax gene invalidation was performed as previously described. The invalidations of the $b c l-2, D n m t 1, D n m t 3 a$ and/or Dnmt3b genes were performed by using the shRNA method and according to the manufacturer's instructions (Sigma, Lyon, France)

\section{Conflict of interest}

The authors declare no conflict of interest.

Acknowledgements. We thank to the Neurosurgery department of the Hôpital $\mathrm{G}$ and R Laennec, CHU Nantes. This work was supported by a grant from the 'Association pour la Recherche contre le Cancer' (ARC\#3907 and ARC\#1020). EH was supported by a fellowship from INCa.

1. Hanahan D, Weinberg R. The hallmarks of cancer. Cell 2000; 100: 57-70.

2. Gopisetty G, Ramachandran K, Singal R. DNA methylation and apoptosis. Mol Immunol 2006; 43: 1729-1740.

3. Estève $\mathrm{P}$, Chin $\mathrm{H}$, Pradhan $\mathrm{S}$. Molecular mechanisms of transactivation and doxorubicinmediated repression of survivin gene in cancer cells. J Biol Chem 2007; 282: 2616-2625.

4. Wang S, Liu N, Wang J, Bai H, Mao L. Critical role of deltaDNMT3B4/2 in regulating RASSF1A promoter-specific DNA methylation in non-small cell lung cancer. Chin Med $J$ 2008; 121: 1712-1721.

5. Hervouet E, Debien E, Charbord J, Menanteau J, Vallette FM, Cartron PF. Folate supplementation: a tool to limit the aggressiveness of gliomas via the re-methylation of DNA repeat element and genes governing apoptosis and proliferation. Clin Cancer Res 2009; 15: 3519-3529.

6. Zukiel R, Nowak S, Barciszewska A, Gawronska I, Keith G, Barciszewska M. A simple epigenetic method for the diagnosis and classification of brain tumors. Mol Cancer Res 2004; 2: 196-202.

7. Cartron PF, Oliver L, Martin S, Moreau C, LeCabellec MT, Jezequel P et al. The expression of a new variant of the pro-apoptotic molecule Bax, Baxpsi, is correlated with an increased survival of glioblastoma multiforme patients. Hum Mol Genet 2002; 11: 675-687.

8. Cartron PF, Juin P, Oliver L, Martin S, Meflah K, Vallette F. Nonredundant role of Bax and Bak in Bid-mediated apoptosis. Mol Cell Biol 2003; 23: 4701-4712.

9. Martinez R, Setien F, Voelter C, Casado S, Quesada M, Schackert G et al. CpG island promoter hypermethylation of the pro-apoptotic gene caspase- 8 is a common hallmark of relapsed glioblastoma multiforme. Carcinogenesis 2007; 28: 1264-1268.

10. Gao Y, Guan M, Su B, Liu W, Xu M, Lu Y. Hypermethylation of the RASSF1A gene in gliomas. Clin Chim Acta 2004; 349: 173-179.

11. Yu J, Zhang H, Gu J, Lin S, Li J, Lu W et al. Methylation profiles of thirty four promoter-CpG islands and concordant methylation behaviours of sixteen genes that may contribute to carcinogenesis of astrocytoma. BMC Cancer 2004; 4: 65.

12. Stone A, Bobo W, Brat D, Devi N, Van Meir E, Vertino P. Aberrant methylation and down-regulation of TMS1/ASC in human glioblastoma. Am J Pathol 2004; 165 : 1151-1161.

13. Nakamura M, Ishida E, Shimada K, Nakase H, Sakaki T, Konishi N. Frequent HRK inactivation associated with low apoptotic index in secondary glioblastomas. Acta Neuropathol 2005; 110: 402-410.

14. McConnell B, Vertino P. TMS1/ASC: the cancer connection. Apoptosis 2004; 9: 5-18.

15. Juin $\mathrm{P}$, Cartron $\mathrm{P}$, Vallette $\mathrm{F}$. Activation of $\mathrm{Bax}$ by $\mathrm{BH} 3$ domains during apoptosis: the unfolding of a deadly plot. Cell Cycle 2005; 4: 637-642.

16. Inohara N, Ding L, Chen S, Núñez G. Harakiri, a novel regulator of cell death, encodes a protein that activates apoptosis and interacts selectively with survival-promoting proteins $\mathrm{Bcl}-2$ and $\mathrm{Bcl}-\mathrm{X}(\mathrm{L})$. EMBO J 1997; 16: 1686-1694

17. Lalier $L$, Cartron $P$, Juin $P$, Nedelkina $S$, Manon $S$, Bechinger $B$ et al. Bax activation and mitochondrial insertion during apoptosis. Apoptosis 2007; 12: 887-896.

18. Shin S, Sung B, Cho Y, Kim H, Ha N, Hwang J et al. An anti-apoptotic protein human survivin is a direct inhibitor of caspase-3 and -7. Biochemistry 2001; 40: 1117-1123.

19. Liston P, Fong W, Kelly N, Toji S, Miyazaki T, Conte D et al. Identification of XAF1 as an antagonist of XIAP anti-caspase activity. Nat Cell Biol 2001; 3: 128-133.

20. Li L, Dahiya R. MethPrimer: designing primers for methylation PCRs. Bioinformatics 2002; 18: $1427-1431$.

21. Estève $\mathrm{P}$, Chin $\mathrm{H}$, Pradhan $\mathrm{S}$. Human maintenance DNA (cytosine-5)-methyltransferase and p53 modulate expression of p53-repressed promoters. Proc Natl Acad Sci USA 2005; 102: $1000-1005$

22. Fatemi M, Hermann A, Gowher $\mathrm{H}$, Jeltsch A. Dnmt3a and Dnmt1 functionally cooperate during de novo methylation of DNA. Eur J Biochem 2002; 269: 4981-4984.

23. Liang G, Chan M, Tomigahara Y, Tsai Y, Gonzales F, Li E et al. Cooperativity between DNA methyltransferases in the maintenance methylation of repetitive elements. Mol Cell Biol 2002; 22: 480-491.

24. Kim G, Ni J, Kelesoglu N, Roberts R, Pradhan S. Co-operation and communication between the human maintenance and de novo DNA (cytosine-5) methyltransferases. EMBO J 2002; 21: 4183-4195.

25. Rhee I, Bachman KE, Park BH, Jair KW, Yen RW, Schuebel KE et al. DNMT1 and DNMT3b cooperate to silence genes in human cancer cells. Nature 2002; 416: 552-556.

26. Sun L, Huang L, Nguyen P, Bisht K, Bar-Sela G, Ho A et al. DNA methyltransferase 1 and 3B activate BAG-1 expression via recruitment of CTCFL/BORIS and modulation of promoter histone methylation. Cancer Res 2008; 68: 2726-2735.

27. Sowiñska A, Jagodzinski P. RNA interference-mediated knockdown of DNMT1 and DNMT3B induces CXCL12 expression in MCF-7 breast cancer and AsPC1 pancreatic carcinoma cell lines. Cancer Lett 2007; 255: 153-159.

28. Stimson K, Vertino P. Methylation-mediated silencing of TMS1/ASC is accompanied by histone hypoacetylation and $\mathrm{CpG}$ island-localized changes in chromatin architecture. J Biol Chem 2002; 277: 4951-4958

29. Deplus R, Brenner C, Burgers WA, Putmans P, Kouzarides T, de Launoit $Y$ et al. Dnmt3L is a transcriptional repressor that recruits histone deacetylase. Nucleic Acids Res 2002; 30: 3831-3838.

30. Conway K, McConnell B, Bowring C, Donald C, Warren S, Vertino P. TMS1, a novel proapoptotic caspase recruitment domain protein, is a target of methylation-induced gene silencing in human breast cancers. Cancer Res 2000; 60: 6236-6242.

31. Babidge W, Butler L, Burton M, Cowled P. Methylation of $\mathrm{CpG}$ sites in exon 2 of the bcl-2 gene occurs in colorectal carcinoma. Anticancer Res 2001; 21: 2809-2814.

32. Hanada M, Delia D, Aiello A, Stadtmauer E, Reed JC. Bcl-2 gene hypomethylation and high-level expression in B-cell chronic lymphocytic leukemia. Blood 1993; 82: 1820-1828.

33. Zou B, Chim C, Zeng H, Leung S, Yang Y, Tu S et al. Correlation between the single-site $\mathrm{CpG}$ methylation and expression silencing of the XAF1 gene in human gastric and colon cancers. Gastroenterology 2006; 131: 1835-1843.

34. Kempkensteffen C, Hinz S, Schrader M, Christoph F, Magheli A, Krause H et al. Gene expression and promoter methylation of the XIAP-associated factor 1 in renal cell carcinomas: correlations with pathology and outcome. Cancer Lett 2007; 254: 227-235.

35. Byun D, Cho K, Ryu B, Lee M, Kang M, Kim H et al. Hypermethylation of XIAP-associated factor 1, a putative tumor suppressor gene from the 17p13.2 locus, in human gastric adenocarcinomas. Cancer Res 2003; 63: 7068-7075.

36. Fong W, Liston P, Rajcan-Separovic E, St Jean M, Craig C, Korneluk R. Expression and genetic analysis of XIAP-associated factor 1 (XAF1) in cancer cell lines. Genomics 2000; 70: $113-122$.

37. Juin P, Pelletier M, Oliver L, Tremblais K, Grégoire M, Meflah $\mathrm{K}$ et al. Induction of a caspase-3-like activity by calcium in normal cytosolic extracts triggers nuclear apoptosis in a cell-free system. J Biol Chem 1998; 273: 17559-17564.

38. Li LC, Dahiya R. Methprimer: designing primers for methylation PCRs. Bioinformatics 2002; 11: 1427-1431.

(c)

Cell Death and Disease is an open-access journal published by Nature Publishing Group. This article is licensed under a Creative Commons Attribution-Noncommercial-No Derivative Works 3.0 License. To view a copy of this license, visit http:// creativecommons.org/licenses/by-nc-nd/3.0/

Supplementary Information accompanies the paper on Cell Death and Disease website (http://www.nature.com/cddis) 